\title{
Possible Mechanisms for Telomere Length Maintenance in Extremely Old People
}

Gutman $D^{1}$, Sharvit $L^{1}$ and Atzmon $\mathbf{G}^{1,2^{*}}$

${ }^{1}$ Department of Human Biology, Faculty of Natural Sciences, University of Haifa, Haifa, Israel

${ }^{2}$ Departments of Medicine and Genetics, Albert Einstein College of medicine, Bronx, New York, USA

"Corresponding author: Gil Atzmon, Departments of Medicine and Genetics, Albert Einstein College of medicine, Bronx, New York, USA, Tel: 78-430-3628; Fax: 78-430-8557; E-mail: gil.atzmon@einstein.yu.edu

Rec date: Oct 31, 2014, Acc date: Nov 1, 2014, Pub date: Nov 3, 2014

Copyright: $\odot 2014$ Gutman D, et al. This is an open-access article distributed under the terms of the Creative Commons Attribution License, which permits unrestricted use, distribution, and reproduction in any medium, provided the original author and source are credited.

Keywords: Telomere; Longevity; HDL

\section{Editorial}

Telomeres, TTAGGG tandem repeats at the ends of chromosomes, become progressively shortened with each replication of cultured human somatic cells [1-4] until a critical length is achieved. Once this length is reached, the cell enters replicative senescence. Telomere shortening has been implicated as a mechanism explaining variations in life expectancy and aging-related diseases. It has been demonstrated in cross-sectional analyses that age affects attrition of telomere length in white blood cells $[2,3]$. Telomere length reflects the cumulative burden of oxidative stress and repeated cell replication $[1,5,6]$, and such oxidative stress may represent the link between telomeres and aging-related diseases in humans.

Probands and their offspring had significantly longer telomeres than offspring's age and gender matched controls. In addition, a possible genetic link for this observance which tied variants of the telomerase gene to telomere length was demonstrated. Furthermore, shorter telomeres were found in subjects with metabolic syndrome, diabetes, hypertension, and impaired cognitive function, while longer telomeres were associated with an improved lipid profile [7].

Living to 100 is a rare phenotype, with prevalence in the general population of 1 in 5,000 individuals [8]. Previous cross-sectional studies have demonstrated that adult telomeres become shorter with age at a relatively constant rate, however those analyses contained relatively few subjects with exceptional longevity $[2,4]$. A similar agerelated decline in telomere length until age 85 was observed, after which telomere length increased significantly [7]. How might we explain the increase in telomere length observed in subjects older than 85 years old? One possibility is that telomere attrition may limit survival in the general population, and that a lack of telomere shortening may have enabled certain people to achieve very old age. These rare individuals may have had longer initial telomere length when they were younger (as reflected in the long telomeres in their offspring [7]), may have had a slower rate of telomere shortening, or both. Another feasible mechanism for this finding is that mechanisms resulting in longer telomeres are activated only at increased age. Increased telomerase activity resulting with increased telomere length might be related to the observation of high HDL levels in offspring compared to control [9]. As speculated by Nofer et al. [10], HDL levels can modulate telomerase activity, resulting in telomere elongation. In addition telomere length was associated with HDL levels [5,7]. We hypothesized that a certain range of HDL levels would activate or deactivate telomerase activity; At higher HDL levels telomerase would be activated, and would be deactivated in lower HDL levels. Since HDL levels decline with age, and the observed HDL levels in centenarians are the same as the controls who are 30 years younger [9], we speculated that centenarians had much higher HDL levels when they were in their 70's - a trait which might be manifested in their offspring. Therefore, telomerase was activated during this period (i.e. 30 years previously) in those who eventually achieved very old age. In contrast, telomerase might have been deactivated in the control group, who has low HDL levels.

Further support for this theory is the observation that among individuals who possessed any of three genotypes (CETP, APOC3 and adiponectin) known to be associated with longevity, telomere length increased with age, whereas those lacking these genotypes showed the opposite trend [11]. It is theoretically possible that heightened telomerase activity in individuals with this favorable genotype resulted not only in a slower rate of telomere attrition, but in increasing telomere length with age. This phenomenon of increasing telomere length with age was previously demonstrated in a small number of subjects in a prospective study of younger adults [12].

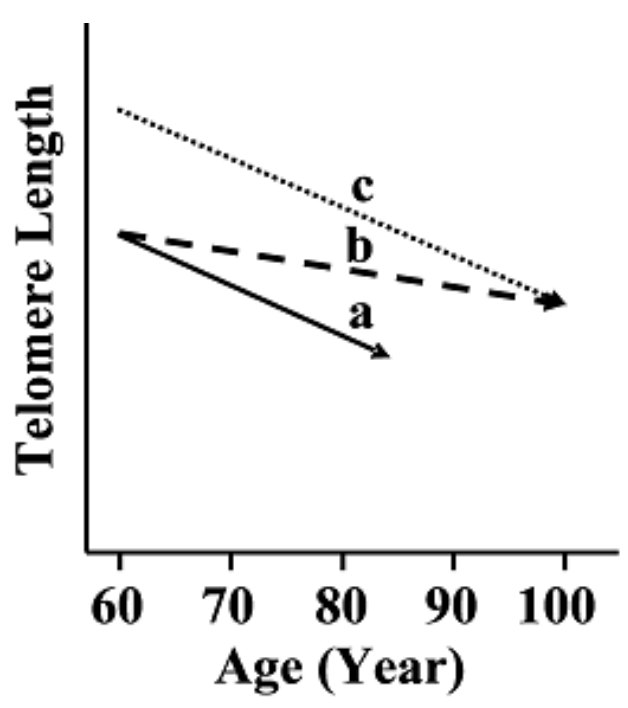

Figure 1: Concept for Telomere length trends according to age. a) Expected attrition rate b) slower attrition rate c) expected attrition rate at higher starting point

Because each of these genes are involved in lipid metabolism, it might also be the case that interaction between telomere attrition and lipid profile affects an individual's potential to survive to very old age.

We propose the following three hypothesizes as possible explanations as to how centenarians might have achieved long 
Citation: Gutman D, Sharvit L, Atzmon G (2014) Possible Mechanisms for Telomere Length Maintenance in Extremely Old People. Hereditary

Page 2 of 2

telomeres (Figure 1): (1) their telomere attrition rate may be slower, (2) the centenarian's attrition rate is "normal" yet they began life with exceptionally long telomeres (as demonstrated by their offspring), (3) the centenarian's attrition rate is "normal" however at a certain age telomerase activity, regulated by HDL levels, becomes activated. That said, it is also possible that a combination of two or more of these processes accounts for the centenarian's long telomeres.

\section{References}

1. Correia-Melo C, Hewitt G, Passos JF (2014) Telomeres, oxidative stress and inflammatory factors: partners in cellular senescence? Longev Healthspan 3.

2. Müezzinler A, Zaineddin AK, Brenner H (2013) A systematic review of leukocyte telomere length and age in adults. Ageing Res Rev 2: 509-519.

3. Sanders JL, Newman AB (2013) Telomere Length in Epidemiology: A Biomarker of Aging, Age-Related Disease, Both, or Neither? Epidemiol Rev .

4. Tedone E, Arosio B, Gussago C, Casati M, Ferri E, et al. (2014) Leukocyte telomere length and prevalence of age-related diseases in semisupercentenarians, centenarians and centenarians' offspring. Exp Gerontol 58C: 90-95.

5. Guo X., Yu M, Kang X, Yin H (2011) mTOR complex 2 activation by reconstituted high-density lipoprotein prevents senescence in circulating angiogenic cells. Arteriosclerosis, thrombosis, and vascular biology 31: 1421-1429.

6. Pu DR, Liu L (2008) HDL slowing down endothelial progenitor cells senescence: a novel anti-atherogenic property of HDL. Med Hypotheses 70: 338-342.

7. Atzmon G, Cho M, Cawthon RM, Budagov T, Katz M, et al. (2010) Evolution in health and medicine Sackler colloquium: Genetic variation in human telomerase is associated with telomere length in Ashkenazi centenarians. Proc Natl Acad Sci U S A 07 Suppl : 1710-177.

8. http://www.census.gov/popclock/

9. Atzmon G et al. (2002) Plasma HDL levels highly correlate with cognitive function in exceptional longevity. The journals of gerontology. Series A, Biological sciences and medical sciences 57: M712-715

10. Nofer JR, Walter M, Assmann G (2005) Current understanding of the role of high-density lipoproteins in atherosclerosis and senescence. Expert Rev Cardiovasc Ther 3: 1071-1086.

11. Atzmon G, Rincon M, Schechter CB, Shuldiner AR, Lipton RB, et al. (2006) Lipoprotein genotype and conserved pathway for exceptional longevity in humans. PLoS Biol 4.

12. Terry DF, Nolan VG, Andersen SL, Perls TT, Cawthon R (2008) Association of longer telomeres with better health in centenarians. J Gerontol A Biol Sci Med Sci 63: 809-812. 